Article

\title{
Influence of the Hamaker Constant on the Value of the Critical Thickness of Foam Films
}

\author{
Dilyana Ivanova-Stancheva $(\mathbb{D}$ \\ Faculty of Natural Sciences, University of Shumen, Shumen 9700, Bulgaria; D.Ivanova@shu.bg; \\ Tel.: +359-896-877-998
}

Received: 31 July 2019; Accepted: 9 September 2019; Published: 10 September 2019

\begin{abstract}
The aim of the present study was to check the influence of the Hamaker constant $(\mathrm{A}(\mathrm{h}))$ on the calculated critical thickness of foam films. It was done by a comparison between the experimental data of the critical thickness and the theoretically obtained data with different values of $(\mathrm{A}(\mathrm{h}))$, which take part in the equations of Radoev, Scheludko, Manev, and Vrij. We calculated the latter for film thicknesses from 15 to $70 \mathrm{~nm}$ based on several equations. We used the experimental data of $h_{\mathrm{cr}}$ (average value of critical thickness and measured once, in the place of a spot appearance) for foam films stabilized by non-ionic surfactant $n$-dodecyl- $\beta$-D-maltoside $\left(C_{12} G_{2}\right)$ or by cationic surfactant n-dodecyl trimethylammonium bromide $\left(\mathrm{C}_{12} \mathrm{TAB}\right)$, as measured by the interferometric method. The foam films were produced from solutions with surfactant concentration at CMC (Critical Micelle Concentration) in the presence of $0.1 \mathrm{M} \mathrm{NaBr}$. For films of $\mathrm{C}_{12} \mathrm{G}_{2}$, the best correspondence was found between measured $h_{c r}$ (in the spot) and calculated ones by the RShM equation using effective $A(h)$ (introduced by Coons et al.) or Vassilieff's values, but with a correction for the added electrolyte. For films stabilized by $\mathrm{C}_{12} \mathrm{TAB}$, a difference between the measured and theoretically calculated $\mathrm{h}_{\mathrm{cr}}$ was found, which was probably due to the nature of the surfactant. Nevertheless, this difference was smallest when calculated $h_{\mathrm{cr}}$ values were obtained with data for Aeff (h) and Vassilieff's $\mathrm{A}(\mathrm{h})$ constant, respectively.
\end{abstract}

Keywords: Hamaker constant; foam films; critical thickness; drainage coefficient; surfactant

\section{Introduction}

Foams need surfactants for their stabilization, but toxicity issues are also important. Thus, sugar-based surfactants are not dangerous for human health. In essence, they are non-ionic and biodegradable surfactants. Moreover, they cannot be affected by the temperature and $\mathrm{pH}$ variations. In addition, they are insensitive to $\mathrm{Ca}$ and $\mathrm{Mg}$ ions in the water. [1-3] provide more information about the sugar-based surfactant $n$-dodecyl- $\beta$-D-maltoside $\left(\mathrm{C}_{12} \mathrm{G}_{2}\right)$, thus indicating that it can replace some of the conventional non-ionic surfactants. Ionic surfactants also have many advantages as well. The cationic surfactant n-dodecyl trimethylammonium bromide $\left(\mathrm{C}_{12} \mathrm{TAB}\right)$ is an antiseptic, anti-bacterial, and fungicidal substance [4]. If $\mathrm{C}_{12} \mathrm{TAB}$ is combined with a non-ionic surfactant, they synergize [5], thus controlling the surface charge and the very electrostatic stabilization [6] of dispersed systems. The kinetic behavior of foam films is important for the foam stability [7]. They often are under non-equilibrium conditions. Of particular importance for the stability of the foam films is their critical thickness $\left(\mathrm{h}_{\mathrm{cr}}\right)$, at which the films either rupture or undergo transition to black stable films, which consist of surfactant bilayers [8]. The critical thickness of the foam films is usually measured interferometrically [9], or can be calculated theoretically [10-12]. Ref. [13,14] reported that the theoretical values of $h_{c r}$ are lower than the measured ones, regardless of the approach used. For this reason, improvements are needed for a correct calculation of film critical thickness. For example, an additional non-DLVO driving force can be accounted for, or more precise calculation of the values of 
the Hamaker function can be made. The first corrections are made for calculating the critical thickness values of films containing single surfactants [14] and their mixtures [15]. According to the literature, in the most cases, the values of the Hamaker constants are taken from a variety of sources, and the choice is not justified. In Ref. [12], the authors compared measured and theoretical predicted $\mathrm{h}_{\mathrm{cr}}$ values from the bounding scaling equations in combination with the theoretical values for the velocity of film thinning, as well as with different values for A(h). Three Hamaker constants-non-retarded, retarded, and effective- $\mathrm{A}_{\text {eff }}(\mathrm{h})$ were used in this study. It was reported that the retarded Hamaker constants calculated by means of the approximate Lifshitz theory underestimate the experimental critical thickness. In addition, the non-retarded Hamaker constant enables closer theoretical predictions of the critical film thickness to the experimental one for films with different radii. It was established that only a part of the foam and emulsion critical thickness measurements are bounded when the effective form of the retarded Hamaker constant is used. Moreover, it was established that the interfacial interactions in flexible thin films are not adequately represented by the retarded Hamaker constant calculated by means of the Lifshitz theory. It is known that the value of the Hamaker constant is of importance for the correlation between the measured and theoretical $h_{\mathrm{cr}}$. From our point of view, the result could be improved if the measured velocity of film thinning is used instead of the theoretically estimated one. The aim of the presented study is in this direction: to compare measured with theoretical calculated $h_{c r}$ values using both different values of $A(h)$ and experimental values for film drainage. In order to make this comparison, a set of the following data was needed:

- Experimental data of the critical thickness (in the place of the black spot formation) for films stabilized by nonionic surfactant n-dodecyl- $\beta$-D-maltoside $\left(C_{12} G_{2}\right)$ and cationic surfactant $\mathrm{n}$-dodecyl trimethylammonium bromide $\left(\mathrm{C}_{12} \mathrm{TAB}\right)$ in the presence of $0.1 \mathrm{M} \mathrm{NaBr}$;

- Values of the Hamaker constant calculated by means of different equations for film thicknesses from 15 to $70 \mathrm{~nm}$;

- Values of critical thickness calculated by Vrij approach and Radoev-Scheludko-Manev (RShM) formula, using the measured drainage coefficients and the calculated values of the Hamaker constant.

In the study, we discuss the correlation between the experimental data of critical thickness and theoretical ones in order to estimate the influence of the Hamaker constant on $\mathrm{h}_{\mathrm{cr}}$.

\section{Materials and Methods}

\subsection{Materials}

The non-ionic surfactant n-dodecyl- $\beta$-D-maltoside $\left(\mathrm{C}_{12} \mathrm{G}_{2}\right.$, purity $\left.>99.5 \%\right)$ was purchased from Glycon (Luckenwalde, Germany). The cationic surfactant dodecyl trimethylammonium bromide $\left(\mathrm{C}_{12} \mathrm{TAB}\right.$, purity $>98 \%$ ) (Fluka, Germany) was provided by Prof. C. Stubenrauch. Sodium bromide $(\mathrm{NaBr})$ was purchased from Sigma-Aldrich (Netherlands) and roasted for $4 \mathrm{~h}$ at $300{ }^{\circ} \mathrm{C}$ to drive off the organic contaminants. All experiments were carried out at temperature $25 \pm 1{ }^{\circ} \mathrm{C}$.

\subsection{Experimental Conditions}

In order to realize the aim of the present study, foam films (with radii from 0.04 to $0.15 \mathrm{~mm}$ ) were obtained from solution with $\mathrm{C}_{12} \mathrm{G}_{2}$ or $\mathrm{C}_{12} \mathrm{TAB}$ in the presence of $0.1 \mathrm{M} \mathrm{NaBr}$ (enough to suppress the electrostatics in the film, e.g., $\Pi_{e l}=0$, which makes it easier to calculate the critical thickness and verify the influence of the $\mathrm{A}(\mathrm{h}))$. The surfactant concentration was equal to critical micelle concentration $(\mathrm{CMC})$, because in this case, the adsorption layers are filled. The solution with $\mathrm{C}_{12} \mathrm{G}_{2}+0.1 \mathrm{M} \mathrm{NaBr}$ has a surfactant concentration of $1.5 \times 10^{-4} \mathrm{M}$ and surface tension of $33.8 \mathrm{mN} / \mathrm{m}$ [16]; solution with $\mathrm{C}_{12} \mathrm{TAB}$ $+0.1 \mathrm{M} \mathrm{NaBr}$ has a surfactant concentration of $4.2 \times 10^{-3} \mathrm{M} \mathrm{[16]} \mathrm{and} \mathrm{surface} \mathrm{tension} \mathrm{of} 35.2 \mathrm{mN} / \mathrm{m}$. 


\subsection{Methods}

Interferometric Method for Thin Liquid Films Investigation

Foam films of different radii (from 0.05 to $0.15 \mathrm{~mm}$ ) were obtained from each solution in a Scheludko-Exerowa glass cell (with radius of the holder $2.15 \mathrm{~mm}$ ) [9]. The film parameters-radius $\left(r_{f}\right)$, film thickness versus time and critical thickness-were obtained by the microscopic interferometric method following a new procedure, employing video recordings of the film evolution, which has been put forward by us [14]. Its virtue is that the film thickness can be determined in purposefully chosen places. The thickness in area, where the critical state is attained, is called " $\mathrm{h}_{\mathrm{cr}}$ in the spot", the thickness in the other two thinnest zones of the film are averaged and written as " $\mathrm{h}_{\mathrm{cr}}$ "; these thicknesses were determined from several consecutive frames within $0.2 \mathrm{~s}$ before the formation of a black spot (the first spot for films stabilized with $\mathrm{C}_{12} \mathrm{TAB}$ ).

The critical thickness was statistically derived by means of a five-layer model of the thin film structure (see Equation (1) in [17]). Each adsorption layer contains two parts: a layer formed by the hydrocarbon chain with a thickness $d_{1}$ and refractive index $n_{1}$, and a layer of the hydrophilic head of the surfactant molecules with a thickness $d_{2}$ and refractive index $n_{2}$. Films containing $C_{12} G_{2}$ have the following values of $n$ and $d: n_{1}=1.42$ (dodecane refractive index); $n_{2}=1.44$ (refractive index of $60 \%$ glucose solution); $d_{2}=1.14 \mathrm{~nm}$ taken from [1], and $d_{1}=0.96 \mathrm{~nm}$ [2]. For films from $C_{12} \mathrm{TAB}$ solution, a three-layer model was used with $\mathrm{n}_{1}=1.42$ and $\mathrm{d}_{1}=0.96 \mathrm{~nm}$.

\subsection{Calculations}

\subsubsection{Film Drainage}

The velocity of film thinning is the first derivative of the average film thickness on the time. [18] used the empirical Equation (1) and as a consequence Equation (2), yielding a linear dependence of $\ln h$ versus the time $t$, thus having slope coefficient, $\alpha$ :

$$
\begin{aligned}
& V=-\frac{d h}{d t}=\alpha h \\
& \ln h=\ln h_{0}-\alpha t
\end{aligned}
$$

where $h_{0}$ is the thickness after a certain time behind the maximum in the kinetic curve; $h$ is the thickness in each moment, analyzed in the same areas used to determine $h_{\mathrm{cr}}$; and $t$ is the thinning time from $h_{0}$ to $h$.

\subsubsection{Critical Thickness}

Applying Scheludko's mechanism, Vrij [10] developed a theory incorporating the total film thinning during the process of its fluctuational destruction. Vrij proposed a procedure for the determination of the $\mathrm{h}_{\mathrm{cr}}$ based on the following graphic dependences: $\tau_{d}=\tau_{d}(h), \tau_{b r}=\tau_{b r}(h)$ and $\tau_{l}=\tau_{l}(h)$, where $\tau_{b r}$ is the time of the film breakage with constant thickness, $\tau_{d}$ is the time of film drainage during the process of its fluctuational destruction, and $\tau_{l}$ is the life time of the thinning film:

$$
\tau_{l}=\left(\tau_{d}+\tau_{b r}\right)_{\min }
$$

From Equation (3), one can estimate the minimal film thickness $\left(h_{\min }\right)$ from its corresponding condition, Equation (4):

$$
\frac{d \tau_{l}}{d h}=\frac{d \tau_{d}}{d h}+\frac{d \tau_{b r}}{d h}=0
$$


The real value of $h_{\mathrm{cr}}$ is obtained from the intersection point of the tangent to the minimum of the curve $\tau_{l}=\tau_{l}(h)$ with the curve $\tau_{d}=\tau_{d}(h)$ (see Figure A1 in Appendix C). The value of $\tau_{b r}$ has been calculated following Equation (5):

$$
\tau_{b r}=\frac{24 \sigma \mu}{h^{3}(d \Pi / d h)^{2}} f
$$

where $\sigma$ is the surface tension, $\mu$ is the bulk viscosity (for water at $25^{\circ} \mathrm{C}$ is $8.9 \times 10^{-4} \mathrm{~N} \mathrm{~s} / \mathrm{m}^{2}$ ), and $f=\frac{1}{2} \ln \left(\frac{2 \Pi^{1 / 2} \sigma h^{2}}{k_{B} T}(2 f)^{1 / 2}\right)$. $\Pi$ is the disjoining pressure, and in accordance with the classical DLVO theory, it incorporates the attractive van der Waals and repulsive electrostatic double-layer interactions. At sufficiently high ionic strength, the electrostatic stabilization of foam film is suppressed, and the value of the electrostatic disjoining pressure becomes equal to zero; then, $\Pi$ coincides with the van der Waals pressure, which is given in the following equation:

$$
\Pi_{v w}=-\frac{A(h)}{6 \pi h^{3}}
$$

where $A(\mathrm{~h})$ is the Hamaker function.

The equation of $\tau_{d}$ can be obtained by any of the equations describing the film thinning. In the present work, $\tau_{d}$ was calculated by Equation (7), which was proposed in [13]:

$$
\tau_{d}=\frac{1}{\alpha} \ln \frac{h_{0}}{h}
$$

This equation gives a possibility of calculating $\tau_{d}$ with the experimental velocity (drainage coefficient $\alpha$ ) instead of by some model equation: it is the merit of this equation. In the present study, Vrij's procedure was employed in order to compare the value of measured $h_{c r}$ (critical thickness) with that predicted by Vrij.

A contrary approach is used from Radoev, Scheludko, and Manev in [11]. Instead of the Reynolds equation, the experimentally velocity of film thinning is used to determine $h_{\mathrm{cr}}$ and its dependence on film size. The authors have shown that, because of the film thickness nonhomogeneity, the way to achieve correct $h_{c r}$ values is to measure the thickness in the thinnest sections of the film. Moreover, the equations for the rate of film thinning used in the theories of the critical thickness are for a model film with plane-parallel and tangentially immobile surfaces. Taking into account the deficiency of the model, Radoev, Scheludko, and Manev (RShM) proposed a new equation for the determination of $h_{\mathrm{cr}}$ :

$$
h_{c r}=0.97 \frac{\left(k_{B} T\right)^{1 / 10} K_{v w^{2 / 5}}}{\mu^{1 / 5} \sigma^{3 / 10} V^{1 / 5}}
$$

where $\mathrm{K}_{\mathrm{vw}}$ is the van der Waals Hamaker constant $K_{v w}=\frac{A(h)}{6 \pi}$

If one inserts Equation (1) in Equation (8), the expression presented by Equation (9) is obtained:

$$
h_{c r}=0.98 \frac{\left(k_{B} T\right)^{1 / 12} K_{v w}{ }^{1 / 3}}{\mu^{1 / 6} \sigma^{1 / 4}}\left(\frac{1}{\alpha}\right)^{1 / 6}
$$

This equation gives a possibility of calculating $h_{\mathrm{cr}}$ with the experimental velocity (drainage coefficient $\alpha$ ), and therefore, it is used in the present paper for the calculation of theoretical data for $h_{\mathrm{cr}}$.

\subsubsection{Hamaker Constant}

The equations above depend on the Hamaker constant, which according to Lifshitz theory, depends on the dielectric spectrum of the substances in the film system and its film thickness. For the symmetric configuration of two identical phases $i$ interacting with the medium $j$, the macroscopic theory offers Equation (10): 


$$
A_{H} \equiv A_{i j i}=A_{i j i}^{(v=0)}+A_{i j i}^{(v>0)}
$$

The first term in the right-hand side of Equation (10), $A_{i j i}(\mathrm{v}=0)$, the so-called zero frequency term, expresses the contribution of the orientation and induction interactions. Indeed, these two contributions to the van der Waals force represent electrostatic effects. The last term in Equation (10), $A_{i j i}(\mathrm{v}>0)$, accounts for the dispersion interaction. If the two phases, $i$ and $j$, have comparable densities (as it is for emulsion systems), then $A_{i j i}(\mathrm{v}=0)$ and $A_{i j i}(\mathrm{v}>0)$ are comparable by magnitude. If one of the phases, $i$ or $j$, has low density (gas, vacuum), as a rule, $A_{i j i}(\mathrm{v}>0)>>A_{i j i}(\mathrm{v}=0)$; in this respect, the macroscopic and microscopic theories often give different predictions for the value of $A_{\mathrm{H}}$. Solutions to this equation are described in [12,19-25].

For calculation of the Hamaker constant in $[19,20]$ (thin film 2 with thickness $h$ is obtained between two semi-infinite phases 1 and 3), Equation (11) was used:

$$
\begin{gathered}
A(h)=A^{(v=0)}+\frac{3}{4} k_{B} T \sum_{v=1}^{\infty} \int_{r_{v}}^{\infty} x^{2}\left[\left(\bar{\Delta}_{12}^{-1} \bar{\Delta}_{32}^{-1} e^{x}-1\right)^{-1}+\left(\Delta_{12}^{-1} \Delta_{32}^{-1} e^{x}-1\right)^{-1}\right] d x \\
A^{(v=0)}=\frac{3}{4} k_{B} T \sum_{J=1}^{\infty} \frac{1}{J^{3}}\left(\frac{\varepsilon_{02}-\varepsilon_{01}}{\varepsilon_{02}+\varepsilon_{01}} \cdot \frac{\varepsilon_{02}-\varepsilon_{03}}{\varepsilon_{02}+\varepsilon_{03}}\right)^{J}
\end{gathered}
$$

where $\bar{\Delta}_{i j}=\frac{s_{v i} \varepsilon_{j}-x \varepsilon_{i}}{s_{v i} \varepsilon_{j}+x \varepsilon_{i}} ; \Delta_{i j}=\frac{s_{v i}-x}{s_{v i}+x} ; s_{v i}=\left[x^{2}+\left(\varepsilon_{i} / \varepsilon_{j}-1\right) r_{v}^{2}\right]^{1 / 2} ; \mathrm{j}=$ phase 1,$3 ; \mathrm{i}=2 ; r_{v}=\frac{2 \xi_{v} h \varepsilon_{j}^{1 / 2}}{c}$; $\xi_{v}=\frac{4 \pi^{2} k_{B} T}{h_{p}}$, $\mathrm{x}$ is the integration variable, $\varepsilon \equiv \varepsilon(i \xi)$ is the dependence of dielectric constant on imaginary frequencies; $T$ is the absolute temperature; $k_{\mathrm{B}}$ is the Boltzman's constant $\left(1.381 \times 10^{-23} \mathrm{~J} / \mathrm{K}\right), \mathrm{h}_{\mathrm{p}}$ is the Planck's constant $\left(6.626 \times 10^{-34} \mathrm{Js}\right)$, and $\mathrm{c}$ is the speed of light $\left(3.0 \times 10^{8} \mathrm{~m} / \mathrm{s}\right)$. The authors of $[19,20]$ computed results for $A(h)$ from the exact expression in Equation (11), and the most simple approximate analytical expression was presented. The obtained results are for $\mathrm{A}(\mathrm{h})$ at different film thickness for the systems: free water film (air-water-air), mica-vacuum-mica, polystyrene-water-polystyrene, and air-tetradecane-corundum with an error of up to $1 \%$. The used systems are without electrolytes, and the Debye screening effect is ignored.

In [12,22], the non-retarded Hamaker constant is presented by Equation (13):

$$
A_{\text {Hnon-ret }}=\frac{3}{4} k_{B} T\left(\frac{\varepsilon_{i}-\varepsilon_{j}}{\varepsilon_{i}+\varepsilon_{j}}\right)^{2}+\frac{3 h_{p} v_{e}}{16 \sqrt{2}} \frac{\left(n_{i}^{2}-n_{j}^{2}\right)^{2}}{\left(n_{i}^{2}+n_{j}^{2}\right)^{3 / 2}}
$$

where $\varepsilon_{i}$ and $\varepsilon_{j}$ are the dielectric constants of phases $i$ (air) and $j(w a t e r) ; n_{i}$ and $n_{j}$ are the respective refractive indices for visible light; and, as usual, $v_{e}$ is the main electronic absorption frequency, which is $\approx 3.0 \times 10^{15} \mathrm{~Hz}$ for water and most organic liquids.

Various expressions have been proposed to account for the electromagnetic retardation effect in the Hamaker constant; Russel et al. derived one convenient formula for the case of symmetric films [23]:

$$
A_{i j i}^{(v>0)}=\frac{3 h_{p} v_{e}}{4 \pi} \frac{\left(n_{i}^{2}-n_{j}^{2}\right)^{2}}{\left(n_{i}^{2}+n_{j}^{2}\right)^{3 / 2}} \int_{0}^{\infty} \frac{(1+2 \tilde{h} z) \exp (-2 \tilde{h} z)}{\left(1+2 z^{2}\right)^{2}} d z
$$

where $\widetilde{h}$ is the dimensionless thickness, and is defined by the expression:

$$
\widetilde{h}=n_{j}\left(n_{i}^{2}+n_{j}^{2}\right)^{1 / 2} \frac{2 \pi v_{e} h}{c}
$$

where the integral in Equation (14) is to be solved numerically; for estimates, one can use the approximate interpolating formula:

$$
\int_{0}^{\infty} \frac{(1+2 \widetilde{h} z) \exp (-2 \widetilde{h} z)}{\left(1+2 z^{2}\right)^{2}} d z \approx\left[1+\left(\frac{\pi \widetilde{h}}{4 \sqrt{2}}\right)^{3 / 2}\right]^{-2 / 3}
$$


When the film's core is polar (e.g., water), salts are added to suppress the electrostatic repulsion between the film surfaces. The presence of ions in the film screens the non-retarded term, in the case when the film thickness is significantly larger than the Debye length. The screening effect occurs when electrolytes are present in the substance $j$ (i.e., the film material) by the included Debye length to the non-retarded term given in $[12,21,22]$ by Equation (17).

$$
A_{\text {ret }}(h)=\frac{3}{4} k_{B} T\left(\frac{\varepsilon_{i}-\varepsilon_{j}}{\varepsilon_{i}+\varepsilon_{j}}\right)^{2}(2 \kappa h) e^{-2 \kappa h}+\frac{3 h_{p} v_{e}}{16 \sqrt{2}} \frac{\left(n_{i}^{2}-n_{j}^{2}\right)^{2}}{\left(n_{i}^{2}+n_{j}^{2}\right)^{3 / 2}}\left[1+\left(\frac{\pi \widetilde{h}}{4 \sqrt{2}}\right)^{3 / 2}\right]^{-2 / 3}
$$

where $\mathrm{K}^{-1}=\frac{0.304}{\sqrt{I}}$ is the Debye length in nm (for symmetrical monovalent electrolyte) at $25^{\circ} \mathrm{C}$, and $I$ is the ionic strength of the solution in $\mathrm{mol} / \mathrm{L}$.

In line with Lifshitz theory, the Hamaker constant drops when the films become thicker because of the retardation effects. Nevertheless, the destabilizing effect of the dependence film thickness on the Hamaker constant requires additional attention [12]. The authors of ref. [12] operate with Aeff(h) and suggest the following equation:

$$
A_{e f f}(h)=\left\{1-\frac{1}{3}\left[\left(\frac{d A}{d h}\right)\left(\frac{h}{A(h)}\right)\right]\right\} A(h)
$$

In addition, they show that the film thickness-the Hamaker function dependence (i.e., the term in the square brackets in Equation (18)) depends on the film thickness as well as on the film substance core, but does not fall below -1 for benzene, aniline, chlorobenzene, and aqueous films. The effective Hamaker constant used in [12] was taken as the value of the retarded Hamaker constant at the experimentally measured critical film thickness with an approximate film thickness dependency contribution of -1 .

$$
A_{e f f}(h) \approx \frac{4}{3} A(h)
$$

The authors from [24] have modeled the energy of a van der Waals interaction versus separation distance between a bubble and a particle across salt solution by Lifshitz theory. They report that only the dispersion interaction is important for the modeling of the bubble-particle attachment. In addition, only the refractive index of a mineral particle, which is available in the literature, is sufficient to predict the van der Waals interactions. [25] adapts the van der Waals interaction in foam films:

$$
A(h)_{\text {byNguyen.et.al. }}=(1+2 \kappa h) e^{-2 \kappa h} A_{0}+\frac{3 h_{p} v_{e}}{16 \sqrt{2}} \frac{\left(n_{i}^{2}-n_{j}^{2}\right)^{2}}{\left(n_{i}^{2}+n_{j}^{2}\right)^{1.5}} \times\left\{1+\left(\frac{h}{\lambda}\right)^{q}\right\}^{1 / q}
$$

where $A_{0}=\left(3 k_{B} T / 4\right) \sum_{J=1}^{\infty}\left(1-\varepsilon_{j} / 1+\varepsilon_{j}\right)^{2 J} / J^{3}$. For foam films, $\varepsilon_{j}=80$, and the infinite sum in the equation for $\mathrm{A}_{0}$ is equal to 1.444 , giving $A_{0}=1.083 k_{B} T ; n_{j}^{2}=1.887$ is the square of the characteristic refractive index of the film (water) in the UV region, $q=1.185$; and the characteristic wavelength, $\lambda$, is measured in the units of length and described by:

$$
\lambda \equiv\left(c / \pi^{2} \omega\right) \sqrt{2 /\left(n_{i}^{2}+n_{j}^{2}\right)}=5.59 n m
$$

where $c$ is the speed of light, and $\omega$ is converted to the unit of $1 / \mathrm{s}$ by dividing by $2 \pi$.

In the presented study the $h_{\mathrm{cr}}$ values are calculated by the Vrij approach (see Figure A1, Appendix C) and by the Radoev-Scheludko-Manev (RShM) Equation (9), which is based on the drainage coefficient $\alpha$ (see Appendix B in combination with data for the Hamaker constant). The latter is calculated with Equation (13) ( $\mathrm{A}_{\mathrm{H}}$ non-ret), Equation (17) (Aret(h)), Equation (19) (Aeff(h)), and Equation (20) (A(h) by Nguyen et al.), and with data calculated by Vassilieff [19]. 


\section{Results and Discussion}

\subsection{Hamaker Constant}

The Hamaker constant for film thicknesses from 15 to $70 \mathrm{~nm}$ was calculated using the discussed equations. In Figure 1, our calculated values for the Hamaker function of thickness are presented, taking into account the Debye screening effect, for two concentrations of monovalent electrolyte: $0.1 \mathrm{M}$ (markers) and 0.001 M (lines), respectively. For the non-retarded Hamaker constant, Equation (13) obtained $3.37 \times 10^{-20} \mathrm{~J}$. The Vassiliev's values for $\mathrm{A}(\mathrm{h})$ (computed exact result by Equation (11) for a free foam film) taken from $[19,20]$ are for systems without electrolytes; this is why we introduced a correlation term that took into account the Debye screening effect. $A_{\text {ret }}(\mathrm{h})$ was calculated by Equation $(17), A_{\mathrm{eff}}(\mathrm{h})$ was calculated by Equation (19) in combination with Equation (17), and Nguyen et al. calculated $A(\mathrm{~h})$ by Equation (20). The values are systematized in Table A1 in Appendix A. From Figure 1, we can conclude that:

- Vassilieff's values of the Hamaker constant are very close to the values of $\mathrm{A}_{\mathrm{eff}}(\mathrm{h})$ (introduced by Coons et al.);

- $\quad$ The calculated values of the Hamaker constant by Nguyen are the lowest, probably because the authors model an expression for the bubble-particle system and introduce fitting coefficients for this system;

- There is no significant difference between the calculated values of $\mathrm{A}(\mathrm{h})$ at the two ionic strengths.

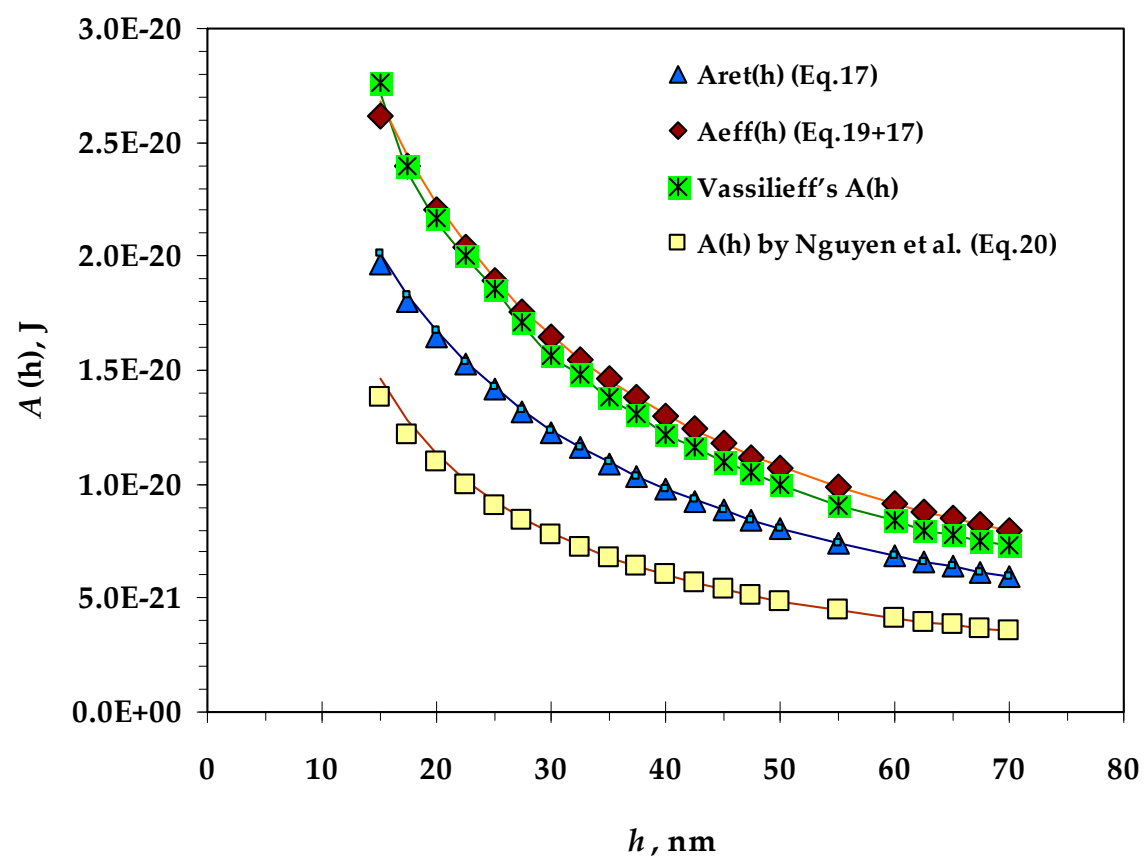

Figure 1. Comparison of the calculated data of Hamaker function by different equations at two ionic strengths $0.1 \mathrm{M}$ (markers) and 0.001 M (lines) for monovalent electrolytes.

3.2. Comparison between Measured and Calculated Critical Thickness Values for Foam Films Stabilized by Nonionic n-Dodecyl- $\beta$-D-maltoside $\left(C_{12} G_{2}\right)$ and by Cationic Surfactant $n$-Dodecyl Trimethyl Ammonium Bromide $\left(C_{12} T A B\right)$

In order to realize the aim of the present study, experiments with foam films (with different radii) from solutions of non-ionic $C_{12} G_{2}$ or cationic $C_{12}$ TAB surfactant in the presence of $\mathrm{NaBr}(0.1 \mathrm{M})$ were performed. The films stabilized by $\mathrm{C}_{12} \mathrm{G}_{2}$ transformed spontaneously into NBF (Newton black films), while in those stabilized by $\mathrm{C}_{12} \mathrm{TAB}$, a few black spots formed successively; after merging these spots, the film passed in CBF (Common black film). In Figures 2 and 3, the data of the critical thicknesses are compared, which were obtained in the place where the black spot was formed "exp. data in the 
spot" (for films of $\mathrm{C}_{12} \mathrm{TAB}$, the place of the formation of the first black spot is meant), with the values estimated for other comparatively thin places on the films labeled as "exp. data". It is found that the $\mathrm{h}_{\mathrm{cr}}$ values in the spot are noticeably smaller than the other "exp. data", especially for films with a radii of $0.15 \mathrm{~mm}$. Furthermore, it is shown that the $h_{\mathrm{cr}}$ value in the spot does not depend on the film radius being greater than about $0.10 \mathrm{~mm}$. These findings permit us to conclude that the value of the film's critical thickness should be averaged from the thickness values determined in many different places of the film during its critical phase.
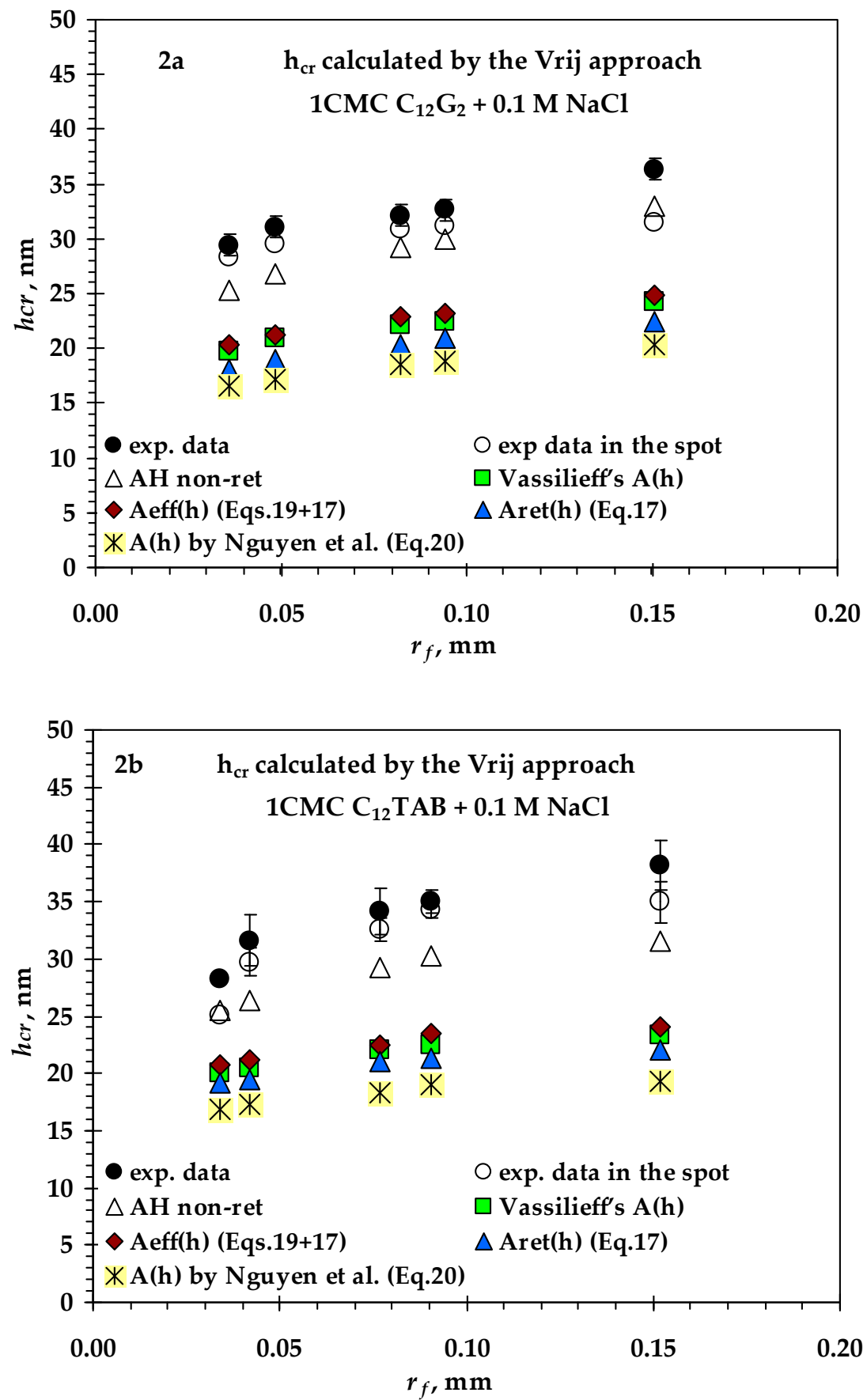

Figure 2. Measured and calculated critical thickness $\left(h_{\mathrm{cr}}\right)$ by Frij approach with different values of A(h), for foam films from solutions with (a) $n$-dodecyl- $\beta$-D-maltoside $\left(C_{12} G_{2}\right)$ or (b) cationic surfactant $\mathrm{n}$-dodecyl trimethylammonium bromide $\left(\mathrm{C}_{12} \mathrm{TAB}\right)$ at $1.0 \mathrm{CMC}$, in the presence of $0.1 \mathrm{M} \mathrm{NaBr}$. 

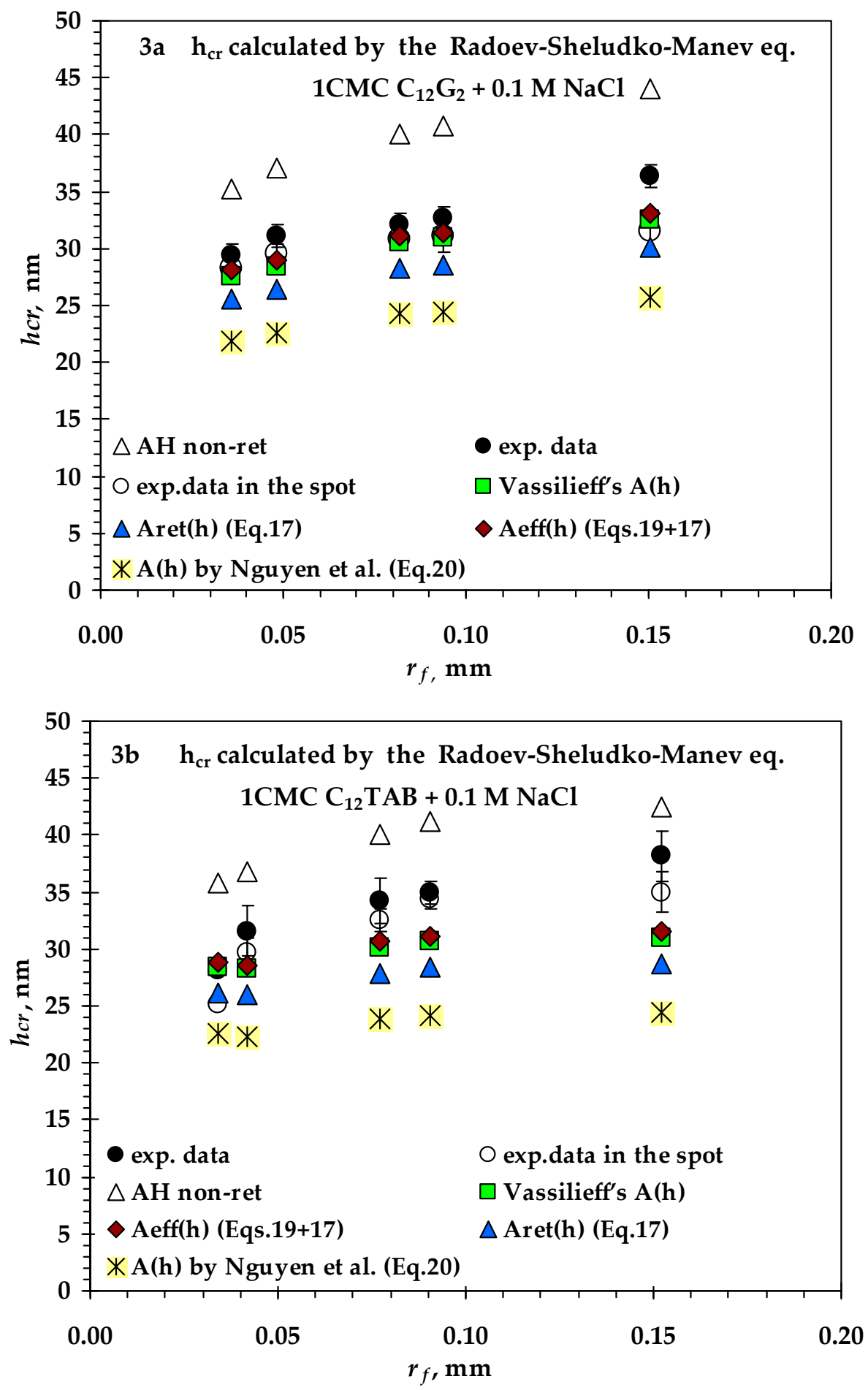

Figure 3. Measured and calculated critical thickness $\left(h_{\mathrm{cr}}\right)$ by Radoev-Scheludko-Manev equation with different values of $A(h)$, for foam films from solutions with (a) $C_{12} G_{2}$ or (b) $C_{12}$ TAB at 1.0 CMC (critical micelle concentration), in the presence of $0.1 \mathrm{M} \mathrm{NaBr}$.

According to the aim of the present study, the measured $h_{c r}$ must be compared to the calculated ones. For this purpose, the film critical thickness was calculated in two different ways: by the Vrij approach (see Figure A1, Appendix C) and by RShM (Equation (9)) in combination with the experimentally determined drainage coefficient $(\alpha)$ (data for $\alpha$ are given in Appendix B) and with different values of $A(h)$. 
Figure 2a,b present experimental and calculated data for $h_{c r}$ in films from solution with $C_{12} G_{2}$ (a) and $\mathrm{C}_{12} \mathrm{TAB}(\mathrm{b})$ at $\mathrm{CMC}$ in the presence of $0.1 \mathrm{M} \mathrm{NaBr}$. The values of calculated $\mathrm{h}_{\mathrm{cr}}$ in Figure $2 \mathrm{a}, \mathrm{b}$ are obtained only by the Vrij approach. It is seen that for all studied radii, instead of used surfactants, the measured $h_{\mathrm{cr}}$ values are higher than the calculated ones. A closer look at data in this figure reveals a correlation only between the measured and calculated $h_{\mathrm{cr}}$ values obtained by the non-retarded Hamaker constant (Equation (13)).

In Figure $3 a, b$ experimental and calculated $h_{c r}$ data are presented, again; this time, they were obtained by the Radoev-Scheludko-Manev equation (Equation (9)). From Figure 3a, it is clear that the measured values of $h_{\mathrm{cr}}$ and $h_{\mathrm{cr}}$ in the spot for films stabilized with $\mathrm{C}_{12} \mathrm{G}_{2}$ have the best correspondence with those calculated by the effective Hamaker constant $\left(\mathrm{A}_{\text {eff }}(h)\right)$ and by the Vassilieff values. The calculated $h_{\mathrm{cr}}$ values for films with radii bigger than $0.10 \mathrm{~mm}$ do not depend on the film radius; this is in good accordance with the same trend obtained for experimental values of $h_{\mathrm{cr}}$ in the spot. It is seen that the average $h_{\mathrm{cr}}$ is higher than $h_{\mathrm{cr}}$ in the spot, and also higher than the calculated $h_{\mathrm{cr}}$ at $0.15-\mathrm{mm}$ film radii. This was attributed to the non-homogeneity of film thickness, which significantly increases with the increasing of the film size.

From Figure $3 \mathrm{~b}$, the same tendency can be seen; predicted $h_{\mathrm{cr}}$ values by using the effective Hamaker constant or by Vassilieff's A(h) are in good agreement with the measured critical thickness, but only for films with small radii. For films with radii up $0.08 \mathrm{~mm}$, the experimental hor values are higher than the calculated ones. This finding can be associated with the non-homogeneity in the film thickness and with the ionic nature of $\mathrm{C}_{12} \mathrm{TAB}$, and consequently, with the appearance of a streaming potential [26,27]. The streaming potential, which was suggested in [26], is due to the dynamic effects originating from the electrical double layer. During the film drainage, the charged liquid from the double layer moves toward the periphery. However, to keep electroneutrality, reverse fluxes arise and bring the charges back; thus, a streaming potential is created. Reynolds explained the slower experimental film thinning compared to predicted ones by this model in [27]. The streaming potential leads to a slight deceleration in the film thinning, which presumes larger hor values.

The RShM formula in combination with the A(h) value (calculated by Nguen et al. in Equation (20)) yields low values of $h_{c r}$, which is related to the coefficients in Equation (20) being calculated for the bubble-particle system.

\section{Conclusions}

The present work checks the influence of the Hamaker constant $(\mathrm{A}(\mathrm{h}))$ on the calculated critical thickness of foam fims produced from solutions of non-ionic surfactant $C_{12} G_{2}$ and ionic $C_{12} T A B$ at $\mathrm{CMC}$ in the presence of $0.1 \mathrm{M} \mathrm{NaBr}$. It is shown that the values of calculated $h_{\mathrm{cr}}$ with Vrij's approach demonstrate a good correlation with experimental data only when the non-retarded Hamaker constant is used (see Figure 2a,b). The analysis demonstrates the best correspondence between the experimental and calculated data for $h_{\mathrm{cr}}$, by Radoev-Scheludko-Manev equation, when Aeff(h) and Vassilieff's values of $A(h)$ are employed (see Figure $3 a, b)$. The higher values of the measured critical thickness for films of $C_{12} T A B$ than the calculated ones are related with the ionic nature of $C_{12} T A B$, and consequently, with the appearance of a streaming potential.

Funding: This research received no external funding.

Acknowledgments: This work is supported by the Bulgarian Ministry of Education and Science under the National Program for Research "Young Scientists and Postdoctoral Students".

Conflicts of Interest: The author declares no conflict of interest. 


\section{Appendix A}

Table A1. Data of Hamaker constants obtained by different equations, taking into account the Debye screening effect, for the presence of $0.1 \mathrm{M}$ monovalent electrolyte.

\begin{tabular}{ccccc}
\hline & \multicolumn{4}{c}{$\mathbf{A}_{\mathbf{H}}$ Retarded $\times \mathbf{1 0}^{\mathbf{2 0}} \mathbf{,} \mathbf{~}$} \\
\cline { 2 - 5 } $\mathbf{h}, \mathbf{n m}$ & Vassilieff's A(h) & $\begin{array}{c}\text { Aret(h) } \\
\text { (by Equation (17)) }\end{array}$ & $\begin{array}{c}\text { Aeff (h) } \\
\text { (by Equation (19) }+\mathbf{( 1 7 ) )}\end{array}$ & $\begin{array}{c}\text { A(h) by Nguyen et al. } \\
\text { (Equation (20)) }\end{array}$ \\
\hline 70.0 & 0.73 & 0.59 & 0.79 & 0.36 \\
67.5 & 0.75 & 0.62 & 0.82 & 0.37 \\
65.0 & 0.78 & 0.64 & 0.85 & 0.38 \\
62.5 & 0.80 & 0.66 & 0.88 & 0.40 \\
60.0 & 0.84 & 0.68 & 0.91 & 0.41 \\
55.0 & 0.91 & 0.74 & 0.99 & 0.45 \\
50.0 & 1.00 & 0.80 & 1.07 & 0.49 \\
47.5 & 1.05 & 0.84 & 1.12 & 0.51 \\
45.0 & 1.10 & 0.88 & 1.18 & 0.54 \\
42.5 & 1.16 & 0.93 & 1.24 & 0.57 \\
40.0 & 1.22 & 0.98 & 1.30 & 0.60 \\
37.5 & 1.31 & 1.03 & 1.38 & 0.64 \\
35.0 & 1.38 & 1.09 & 1.46 & 0.68 \\
32.5 & 1.48 & 1.16 & 1.55 & 0.72 \\
30.0 & 1.56 & 1.23 & 1.65 & 0.78 \\
27.5 & 1.71 & 1.32 & 1.76 & 0.84 \\
25.0 & 1.86 & 1.42 & 1.89 & 0.91 \\
22.5 & 2.00 & 1.53 & 2.04 & 1.00 \\
20.0 & 2.17 & 1.65 & 2.20 & 1.10 \\
17.5 & 2.40 & 1.80 & 2.40 & 1.22 \\
15.0 & 2.76 & 1.97 & 2.62 & \\
\hline
\end{tabular}

\section{Appendix B}

Table A2. Data of experimentally determined drainage coefficient for films obtained from two solutions, $1.5 \times 10^{-4} \mathrm{M} \mathrm{C}_{12} \mathrm{G}_{2}$ with $0.1 \mathrm{M} \mathrm{NaBr}$ and $4.2 \times 10^{-3} \mathrm{M} \mathrm{C}_{12} \mathrm{TAB}$ with $0.1 \mathrm{M} \mathrm{NaBr}$.

\begin{tabular}{|c|c|c|c|}
\hline \multicolumn{2}{|c|}{$1.5 \times 10^{-4} \mathrm{M} \mathrm{C}_{12} \mathrm{G}_{2}+0.1 \mathrm{M} \mathrm{NaBr}$} & \multicolumn{2}{|c|}{$4.2 \times 10^{-3} \mathrm{M} \mathrm{C}_{12} \mathrm{TAB}+0.1 \mathrm{M} \mathrm{NaBr}$} \\
\hline Radius, $\mathbf{m m}$ & $\alpha, \mathrm{s}^{-1}$ & Radius, $\mathrm{mm}$ & $\alpha, \mathrm{s}^{-1}$ \\
\hline 0.036 & 0.0430 & 0.034 & 0.0390 \\
\hline 0.048 & 0.0320 & 0.042 & 0.0334 \\
\hline 0.082 & 0.0200 & 0.077 & 0.0199 \\
\hline 0.094 & 0.0180 & 0.090 & 0.0171 \\
\hline 0.151 & 0.0113 & 0.152 & 0.0140 \\
\hline
\end{tabular}

\section{Appendix C}

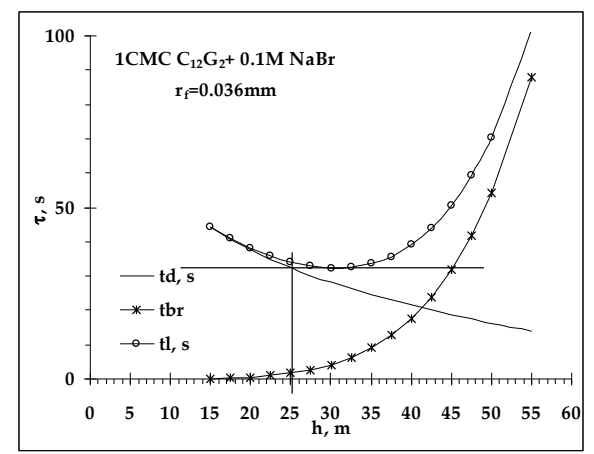

Figure A1. Dependence of the: time of the film drainage during the process of its fluctuation and destruction, including the time of film breakage and the lifetime of the thinning film on the thickness $\left[\tau_{d}=\tau_{d}(h)\right.$ calculated by Equation (7), $\tau_{b r}=\tau_{b r}(h)$ calculated by Equation (5) using the non-retarded Hamaker constant, and $\tau_{l}=\tau_{l}(h)$ calculated similar to a sum from $\tau_{\mathrm{d}}$ and $\tau_{\mathrm{br}}$ ]. 


\section{References}

1. Stubenrauch, C.; Schlarmann, J.; Strey, R. A disjoining pressure study of n-dodecyl-b-D-maltoside foam films. Phys. Chem. Chem. Phys. 2002, 4, 4504-4513. [CrossRef]

2. Muruganathan, R.M.; Krustev, R.; Müller, H.J.; Möhwald, H.; Kolaric, B.; Klitzing, R.V. Foam films stabilized by dodecyl maltoside. 1. Film thickness and free energy of film formation. Langmuir 2004, 20, 6352-6358. [CrossRef] [PubMed]

3. Cristobal, R. (Ed.) Sugar-Based Surfactants; Surfactant Science Series; CRC Press: Boca Raton, FL, USA, 2009; Volume 143.

4. Stubenrauch, C.; Khristov, K. Foams and foam films stabilized by CnTAB: Influence of the chain length and of impurities. J. Colloid Interface Sci. 2005, 286, 710-718. [CrossRef] [PubMed]

5. Patil, S.; Buchavzov, N.; Carey, E.; Stubenrauch, C. Binary mixtures of $\beta$-dodecylmaltoside $\left(\beta-C_{12} G_{2}\right)$ with cationic and non-ionic surfactants: Micelleand surface compositions. Soft Matter 2008, 4, 840-848. [CrossRef]

6. Buchavzov, N.; Stubenrauch, C. A disjoining pressure study of foam films stabilized by mixtures of nonionic and ionic surfactants. Langmuir 2007, 23, 5315-5323. [CrossRef] [PubMed]

7. Petkova, R.; Tcholakovaand, N.S.; Denkov, D. Foamingand Foam Stability for Mixed Polymer-Surfactant Solutions: Effects of Surfactant Typeand Polymer Charge. Langmuir 2012, 28, 4996-5009. [CrossRef] [PubMed]

8. Manev, E.; Scheludko, A.; Exerowa, D. Effect of surfactant concentration on the critical thicknesses of liquid films. Colloid Polym. Sci. 1974, 252, 586-593. [CrossRef]

9. Exerowa, D.; Kruglyakov, P.M. (Eds.) Foam, Foam Films: Theory, Experiment, Application; Marcel Dekker: New York, NY, USA, 1997.

10. Vrij, A. Posible mehanism for spontaneous rupture of thin, free liquid films. Discuss. Faraday Soc. 1966, 42, 23-33. [CrossRef]

11. Radoev, B.; Scheludko, A.; Manev, E. Critical thickness of thin liquid films. Theory and experiment. J. Colloid Interface Sci. 1983, 95, 254-265. [CrossRef]

12. Coons, J.E.; Halley, P.J.; Mc Glashan, S.A.; Tran-Cong, T. Scaling laws for the critical rupture thickness of common thin films. Colloids Surf. A Physicochem. Eng. Asp. 2005, 263, 258-266. [CrossRef]

13. Manev, E.D.; Angarska, J.K. Critical thickness of thin liquid films: Comparison of theory and experiment. Colloids Surf. A Physicochem. Eng. Asp. 2005, 263, 250-257. [CrossRef]

14. Ivanova, D.S.; Angarska, J.K. Drainage and critical thickness of foam films stabilized by n-dodecyl- $\beta$-D-maltoside. Colloids Surf. A Physicochem. Eng. Asp. 2013, 438, 93-103. [CrossRef]

15. Ivanova, D.; Angarska, J.; Manev, E.; Tachev, K. Critical thickness of foam films stabilized by nonionic or ionic surfactants and their mixtures. Colloids Surf. A Physicochem. Eng. Asp. 2017, 519, 34-45. [CrossRef]

16. Ivanova, D.S.; Angarska, Z.K.; Karakashev, S.I.; Manev, E.D. Drainage of foam films stabilized by n-dodecyl- $\beta$-d-maltoside or dodecyl trimethylammonium bromide and their mixtures. Colloids Surf. A Physicochem. Eng. Asp. 2011, 382, 93-101. [CrossRef]

17. Angarska, J.K.; Manev, E.D. Effect of tetrapentyl ammonium bromide adsorption on the electrostatic interactions in aqueous octaethyleneglycol decylether foam films. Colloids Surf. A Physicochem. Eng. Asp. 2003, 223, 73-82. [CrossRef]

18. Manev, E.; Tsekov, R.; Radoev, B. Effect of thickness non-homogeneity on the kinetic behaviour of microscopic foam films. J. Dispers. Sci. Technol. 1997, 18, 769-788. [CrossRef]

19. Vassilieff, C. On the approximate calculation of the disjoining pressure from the Lifshitz theory. Annu. Sofia Univ. 1977/1978, 72, 5-10.

20. Nir, S.; Vassilieff, C. Vander Waals Interactionsin Thin Films. In Thin Liquid Films; Ivanov, M., Dekker, N.Y., Eds.; CRC Press: New York, NY, USA, 1988; pp. 250-256.

21. Israelachvili, J.N. Intermolecular and Surface Forces; Academic Press: San Diego, CA, USA, 1992.

22. Kralchevsky, P.A.; Nagayama, K. Chapter 5: Liquid Films and Interactions between Particle and Surface. In Particles at Fluid Interfaces and Membranes; Kralchevsky, P., Nagayama, K., Eds.; Elsevier: Amsterdam, The Netherlands, 2001; Volume 10, pp. 183-247.

23. Russel, W.B.; Saville, D.A.; Schowalter, W.R. Colloidal Dispersions; Cambridge University Press: Cambridge, UK, 1989.

24. Nguyen, A.V.; Evans, G.M.; Schulze, H.J. Prediction of van der Waalsinteractioninbubbleparticleattachmentinflotation. Int. J. Miner. Process. 2001, 61, 155-169. [CrossRef] 
25. Karakashev, S.I.; Nguyen, A.V. Effect of sodium dodecyl sulphate and dodecanol mixtures on foam film drainage: Examining influence of surface rheology and intermolecular forces. Colloids Surf. A Physicochem. Eng. Asp. 2007, 293, 229-240. [CrossRef]

26. Tsekov, R.; Ivanova, D.; Slavchov, R.; Radoev, B.; Manev, E.; Nguyen, A.; Karakashev, S. Streaming potential effect on the drainage of thin liquid films stabilized by ionic surfactants. Langmuir 2010, 26, 4703-4708. [CrossRef]

27. Angarska, J.K.; Ivanova, D.S.; Manev, E.D. Drainage of foam films stabilized by nonionic, ionic surfactants and their mixtures. Colloids Surf. A Physicochem. Eng. Asp. 2015, 481, 87-99. [CrossRef]

(C) 2019 by the author. Licensee MDPI, Basel, Switzerland. This article is an open access article distributed under the terms and conditions of the Creative Commons Attribution (CC BY) license (http://creativecommons.org/licenses/by/4.0/). 